\title{
Effect of New Suggested Ferroresonance Limiter on the Stability Domain of Chaotic Ferroresonance in the Power Transformer with Linear Core Model
}

\author{
Hamid Radmanesh ${ }^{1}$, Seyed Hamid Fathi ${ }^{2}$, Mehrdad Rostami ${ }^{3}$ \\ ${ }^{1}$ Electrical Engineering Department, Shahid Sattari Aeronautical University of Science and Technology, Tehran, Iran \\ ${ }^{2}$ Electrical Engineering Department, Amirkabir University of Technology (Tehran Polytechnics), Tehran, Iran \\ ${ }^{3}$ Electrical Engineering Department, Shahed University, Tehran, Iran \\ E-mail: \{Hamid.radmanesh, fathi\}@aut.ac.ir, rostami@shahed.ac.ir \\ Received August 14, 2011; revised September 17, 2011; accepted September 29, 2011
}

\begin{abstract}
This paper studies the effect of new suggested ferroresonance limiter on controlling ferroresonance oscillations in the power transformer. It is expected that this limiter generally can control the ferroresonance. For studying these phenomena, at first ferroresonance is introduced and a general modeling approach is given. A simple case of ferroresonance in a three phase transformer is used to illustrate these phenomena. Then, effect of new suggested ferroresonance limiter on the onset of chaotic ferroresonance and control of these oscillations in a power transformer including linear core losses is studied. Simulation is done on a three phase power transformer while one of its phases is opened, and effect of varying input voltage on occurring ferroresonance overvoltage is studied. Results show that connecting the ferroresonance limiter to the transformer exhibits a great controlling effect on the ferroresonance overvoltage. Phase plane diagram, FFT analysis along with bifurcation diagrams are also presented. Significant effect on occurring chaotic ferroresonance, the range of parameter values that may lead to overvoltage and magnitude of ferroresonance overvoltage is obtained, showed and tabulated.
\end{abstract}

Keywords: Linear Core Losses, Chaos, Bifurcation, Ferroresonance Oscillation, Power Transformers, Ferroresonance Limiter

\section{Introduction}

The ferroresonance is typically initiated by saturable magnetizing inductance of a transformer and a capacitive distribution cable or transmission line connected to the transformer. In most practical situations, ferroresonance results in dominated currents, but in some operating "mode", may cause significant high values distorted winding voltage waveform, which is typically referred to as ferroresonance. Although occurrences of the "resonance" occurring does involves a capacitance and an inductance, but there is no definite resonant frequency ferroresonance occurrence for it. In this phenomenon, more than one response is possible for the same set of parameters, and drifts or transients may cause the response to jump from one steady-state response to another one. Its occurrence is more likely to happen in the absence of adequate damping. Research on ferroresonance in transformers has been conducted over the last 80 years.
The word ferroresonance first appeared in the literature in 1920 [1], although papers on resonance in transformers appeared as early as 1907 [2]. Practical interests had been shown was in the 1930s, when it is shown that the use of series capacitors for voltage regulation could cause ferroresonance in distribution systems [3]. Ferroresonant behavior of a $275 \mathrm{kV}$ potential transformer, fed from a sinusoidal supply via circuit breaker grading capacitance, is studied in [4]. The potential transformer ferroresonance from an energy transfer point of view has been presented in [5]. A systematical method for suppressing ferroresonance at neutral-grounded substations has been studied in [6]. A sensitivity study on power transformer ferroresonance of a $400 \mathrm{kV}$ double circuit has been reviewed in [7]. A novel analytical solution to the fundamental ferroresonance has been given in [8]. In that paper, the problem with the traditional excitation characteristic (TEC) of nonlinear inductors has been investigated. The TEC contains harmonic voltages and/or currents. The Stability 
domain calculations of the period-1 ferroresonance have been investigated in [9]. The application of the wavelet transform and MLP neural network for the ferroresonance identification is used in [10]. The impact of the transformer core hysteresis on the stability domain of ferroresonance modes has been studied in [11]. A 2-D finite-element electromagnetic analysis of an autotransformer experiencing ferroresonance is given in [12]. A new modeling of transformers enabling to simulate slow transients more accurate than the existing models in Simulink/MATLAB is presented in [13]. Controlling ferroresonance oscillations in potential transformer considering nonlinear core losses and the circuit breaker shunt resistance effect has been investigated in [14] and [15]. The effect of linear and nonlinear core losses on the onset of chaotic ferroresonance and duration of transient chaos in an autotransformer has been studied in [16]. In current paper, new suggested ferroresonance limiter is used as compact circuit including one resistor, power electronic switch and control circuit for limiting and stabilizing of unstable and high amplitude ferroresonance oscillation. This resistance is connected to the grounding point of the power transformer and during ferroresonance occurrence; power electronic switch is connect the resistor to the transformer via the controlling circuit. In this work, MATLAB program is used to simulate ferroresonance and related phase plane and bifurcation diagrams. The result of the case study confirms that system states, lead to chaos and bifurcation occurs in proposed model. The presence of the ferroresonance limiter tends to clamp the ferroresonance overvoltage. The ferroresonance limiter successfully, reduces the chaotic region for higher exponents. Simulation of system consists of two cases, at first, system modeling of power transformer without connecting ferroresonance limiter and second, power system contains ferroresonance limiter. Finally compare the result of these two cases.

\section{Power System Modeling}

In this section, power transformer is assumed to be connected to the power system while one of the three switches are open and only two phases of it are energized, which produces induced voltage in the open phase. This voltage, back feeds the distribution line. Ferroresonance will occur if the distribution line is highly capacitive. System involves the nonlinear magnetizing reactance of the transformer's open phase and resulted shunt and series capacitance of the distribution line [17]. Figure 1 shows the reduced equivalent thevenin circuit of the power system.

Linear approximation of the peak current of the magnetization reactance can be presented by (1):

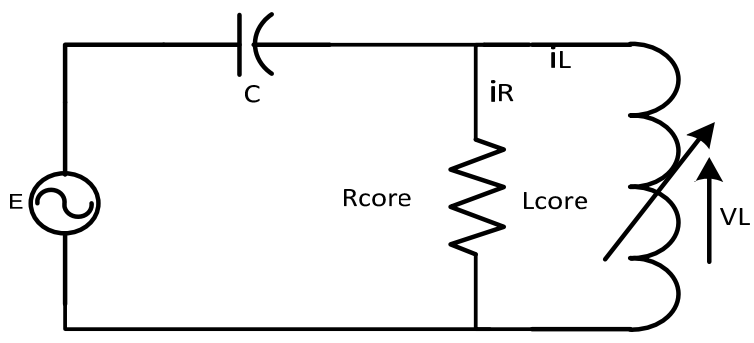

Figure 1. Reduce equivalent thevenin circuit of the power system.

$$
i_{L}=a \varphi
$$

However, for very high currents, the iron core might be saturated where the flux-current characteristic becomes highly nonlinear. The $\varphi-i$ characteristic of the transformer can be demonstrated by the polynomial in (2):

$$
i_{L}=a \varphi+b \varphi^{q}
$$

where " $q$ " is the index of nonlinearity of iron core. The differential equation for the circuit in Figure 1 is derived as follows:

$$
\begin{gathered}
v_{l}=\frac{\mathrm{d} \varphi}{\mathrm{d} t} \\
i_{c}=C \frac{\mathrm{d} v_{c}}{\mathrm{~d} t}, v_{c}=E-v_{l} \\
i_{c}=i_{l}+i_{R} \\
\frac{\mathrm{d} v_{l}}{\mathrm{~d} t}=\frac{\mathrm{d} E}{\mathrm{~d} t}-\frac{1}{R C} \frac{\mathrm{d} \varphi}{\mathrm{d} t}-\frac{1}{C}\left(a \varphi+b \varphi^{q}\right)
\end{gathered}
$$

where $v=\mathrm{d} \varphi / \mathrm{d} t$ and $\omega$ represents the power frequency and $E$ is the peak value of the voltage source as shown in Figure 1. The state-space formulation $\varphi$ and $\mathrm{d} \varphi / \mathrm{d} t$ as state variables is as given below:

$$
\begin{gathered}
\left\{\begin{array}{l}
x_{1}(t)=\varphi \\
x_{2}(t)=\dot{x}_{1}(t) \\
v=\mathrm{d} \varphi / \mathrm{d} t \\
x_{2}(t)=v
\end{array}\right. \\
\dot{x}_{2}(t)=-\frac{1}{R C} x_{2}(t)-\frac{1}{C}\left(a x_{1}(t)+b x_{1}(t)^{q}\right)+\frac{\mathrm{d} E}{\mathrm{~d} t} \\
y(t)=C X(t) \\
y(t)=v(t)=\left[\begin{array}{ll}
0 & 1
\end{array}\right]\left[\begin{array}{l}
x_{1}(t) \\
x_{2}(t)
\end{array}\right] \\
{\left[\begin{array}{l}
u_{1} \\
u_{2}
\end{array}\right]=\left[\begin{array}{l}
0 \\
\frac{\mathrm{d} E}{\mathrm{~d} t}
\end{array}\right]}
\end{gathered}
$$




$$
\begin{gathered}
{\left[\begin{array}{l}
\dot{x}_{1}(t) \\
\dot{x}_{2}(t)
\end{array}\right]=\left[\begin{array}{cc}
0 & 1 \\
\frac{-a-b q}{C} x_{1}(t)^{q-1} & -\frac{1}{R C}
\end{array}\right]\left[\begin{array}{l}
x_{1}(t) \\
x_{2}(t)
\end{array}\right]+\left[\begin{array}{l}
0 \\
1
\end{array}\right] u(11)} \\
\operatorname{det}\left[\begin{array}{cc}
\lambda & -1 \\
\frac{\operatorname{det}[\lambda I-A]=0}{C}(t)^{q-1} & \lambda+\frac{1}{R C}
\end{array}\right]=0 \\
\lambda^{2}+p \lambda+q=0 \lambda_{1,2}=\frac{-p \pm \sqrt{p^{2}-4 q}}{2} \\
p=\frac{(12)}{q} \\
q=\frac{a}{C}
\end{gathered}
$$

\section{Simulation Results of the Power Transformer without Considering Ferroresonance Limiter Effect}

Ferroresonance in three phase systems can involve large power transformers, distribution transformers, or instrument transformers (VT's or CVT's). The general requirements for ferroresonance are an applied or induced source voltage, a saturable magnetizing inductance of a transformer, a capacitance, and little damping. The capacitance can be in the form of capacitance of underground cables or long transmission lines, capacitor banks, coupling capacitances between double circuit lines or in a temporarily-ungrounded system, and voltage grading capacitors in HV circuit breakers. Other possibilities are generator surge capacitors and SVC's in long transmission lines. Due to the multitude of transformer winding and core configurations, various sources of capacitance, and the nonlinearities involved, the scenarios under which ferroresonance oscillations can occur are completely different. System events that may initiate ferroresonance include single-phase switching or fusing, or loss of the system grounding. The ferroresonance circuit in all cases is an applied (or induced) voltage connected to a capacitance in series with a transformer's magnetizing reactance. In this paper, time domain simulations were performed using fourth order Runge-Kutta method and validated against MATLAB SIMULINK. The initial conditions as calculated from steady-state solution of MATLAB are: $\varphi=0,0 ; v_{l}=1.44 \mathrm{pu}$

Simulation is done in two categories, first: power system simulation including transformer linear core losses effect and second: power system simulation considering ferroresonance limiter resistance effect. Typical values for various system parameters considered for simulation without ferroresonance limiter are as given bellowed in Table 1.

In the first step, nonlinear model of the transformer disconnected coil is considered with $q=7$. Figure 2 shows the power system overvoltage considering degree of nonlinearity index $q=7$. This phase plan diagram clearly shows period II oscillation while some extra subharmonic resonances are included in the behavior of the power system. Amplitude of the subharmonic oscillations is reached to 3 p.u, and power spectrum density is shown the existence frequencies in the power system. According to this plot, it is indicate two main frequencies.

Figure 3(a) shows the phase plan diagram of overvoltage on the transformer with $q=11$. It is shown when the degree of $q$ is 11 , amplitude of the overvoltage reaches to 4 p.u. Figure 3(b) shows the power spectrum when input voltage of the power system is 3 p.u. This plot shows the chaotic signal with some subharmonic resonances in it. By referring to this plot, it is shows the overvoltage on transformer is highly nonlinear and chaotic ferroresonance can cause transformer failure. Bifurcation diagram is the best tool for studying the nonlinear dynamic systems. By this tool system behavior can be analyzes in the best view. Figures $\mathbf{4}$ and $\mathbf{5}$ show the bifurcation diagram for $q=7$ and 11 while value of the input voltage is increased from 1 to 8 p.u. In the bifurcation diagram as shown in Figure 4, in point (1) one jump is appeared in the system behavior, in point (2) period V is occurred and when input voltage is reached to $4.5 \mathrm{p} . \mathrm{u}$ as shown with point (3), chaotic ferroresonance is begun, and amplitude of these overvoltage is reached to 3 p.u.

It is also shown when degree of $q$ is increased to 11 ; nonlinear phenomena in the transformer are begun in the low value of the input voltage. It was found that the chaotic behavior begins at a value of $(E=5$ p.u $)$ for $q=7$

Table 1. Power system parameters and per unit value without ferroresonance limiter resistance effect.

\begin{tabular}{ccc}
\hline$Q$ & 7 & 11 \\
\hline Coefficient (a) & 0.0067 & 0.0028 \\
Coefficient (b) & 0.001 & 0.0072 \\
$\omega$ & $377 \mathrm{rad} / \mathrm{sec}$ & $377 \mathrm{rad} / \mathrm{sec}$ \\
& $1 \mathrm{p} . \mathrm{u}$ & $1 \mathrm{p} \cdot \mathrm{u}$ \\
$E$ & $110 / 44 \mathrm{kv}$ & $110 / 44 \mathrm{kv}$ \\
& $1 \mathrm{p.u}$ & $1 \mathrm{p} . \mathrm{u}$ \\
$C$ & $777 \mathrm{nf}$ & $777 \mathrm{nf}$ \\
$S$ & $0.82 \mathrm{p} . \mathrm{u}$ & $0.82 \mathrm{p} . \mathrm{u}$ \\
Initial Condition & $25 \mathrm{MVA}$ & $25 \mathrm{MVA}$ \\
& $0,1.41 \mathrm{p} . \mathrm{u}$ & $0,1.41 \mathrm{p} . \mathrm{u}$ \\
$R_{\text {core }}$ & $750 \Omega$ & $750 \Omega$ \\
& $0.15 \mathrm{p} . \mathrm{u}$ & $0.15 \mathrm{p} . \mathrm{u}$ \\
\hline
\end{tabular}




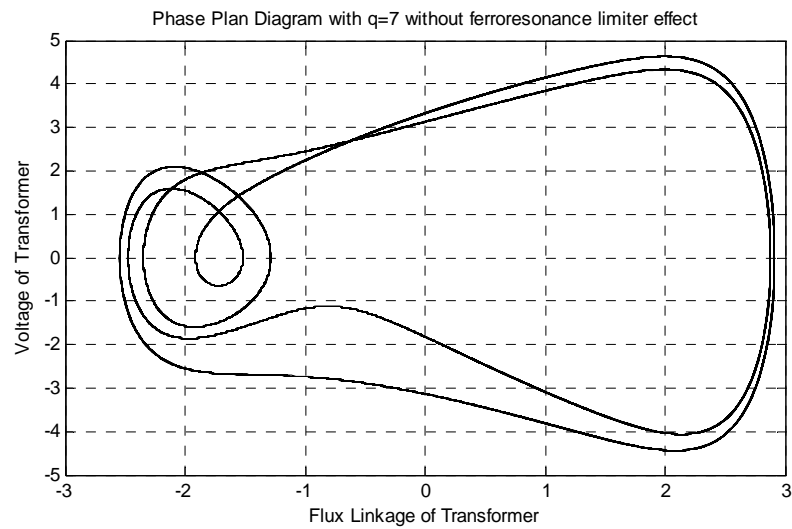

(a)

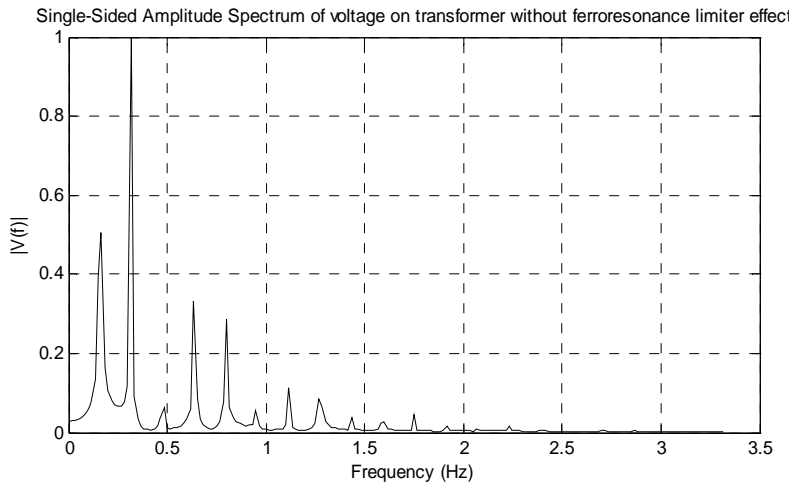

(b)

Figure 2. (a) Phase plan diagram for $q=7$ without considering ferroresonance limiter effect. (b) Single-sided amplitude spectrum without considering ferroresonance limiter effect. Note: in the power spectrum plots, horizontal axis is based on the normalized frequency. It means each $60 \mathrm{~Hz}$ is one unit, so when spectrum shows the 3 units, its actual value is $300 \mathrm{~Hz}$.

and $(E=3$ p.u $)$ for $q=11$ where represents the amplitude of the input voltage of the power system source. Transient chaos settling down to the source frequency and periodic solution was observed for some values of the input voltage as shown in Figure 4.

In Figure 5, input voltage is increased to $8 \mathrm{p} . \mathrm{u}$ and overvoltage on the transformer is analyzed according to the variation of the input voltage. In point (1) one jump is appeared in the system behavior. In point (2) chaotic ferroresonance is appeared. In point (3) chaotic oscillations are changed to the periodic behavior with period III oscillation. Finally, in point (4) system behavior is gone to the chaotic oscillation with period doubling logic. Tendency to chaos exhibited by the system voltage increases while $q$ increases too. Table 2 shows different values of $E$, considered for analyzing the circuit in absence of ferroresonance limiter.

\section{Power System Modeling Considering Ferroresonance Limiter}

The primary purpose of inserting ferroresonance limiter impedance between the star point of a transformer and earth is to limit earth fault current. The value of impedance

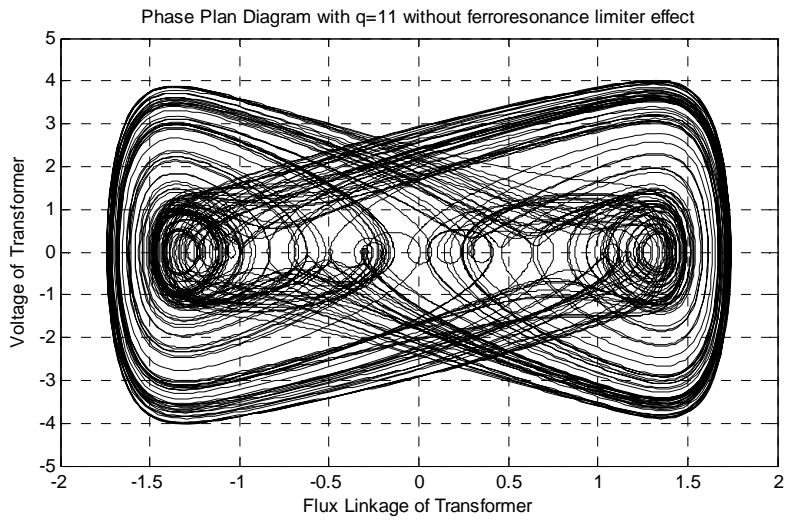

(a)

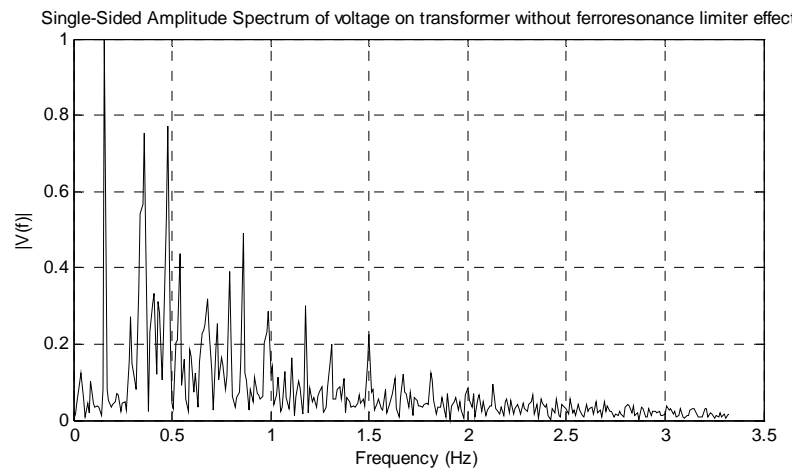

(b)

Figure 3. (a) Phase plan diagram for $q=11$ without considering ferroresonance limiter effect. (b) Single-sided amplitude spectrum without considering ferroresonance limiter effect.

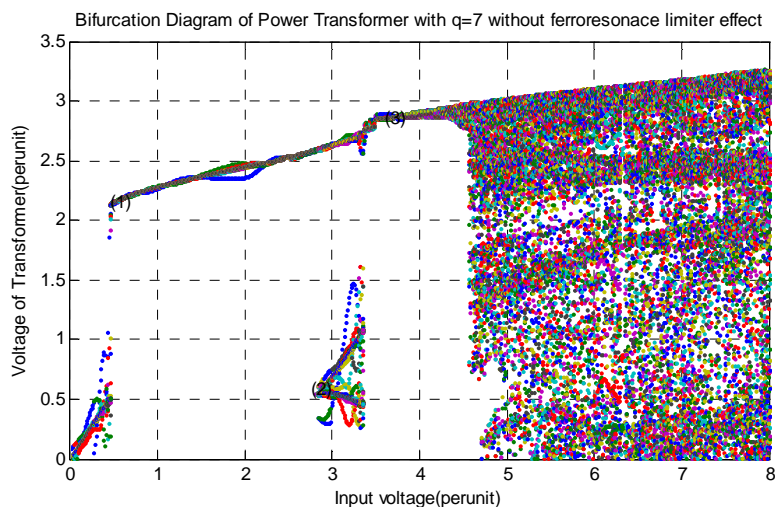

Figure 4. Bifurcation diagram with $q=7$, without connecting ferroresonance limiter. 


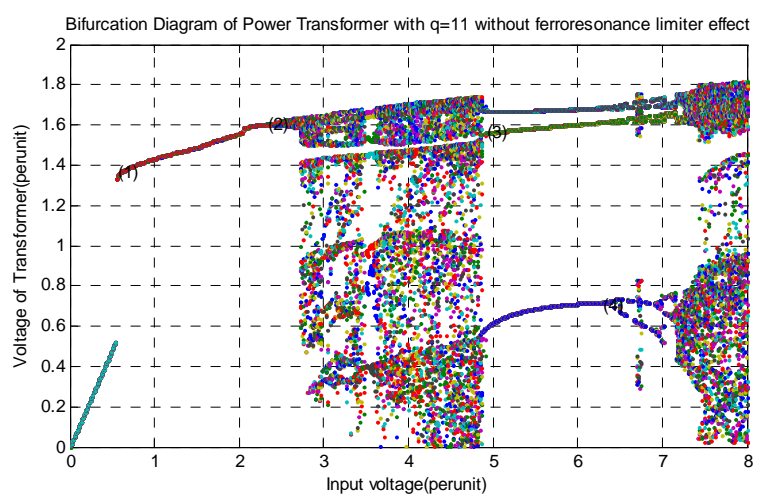

Figure 5. Bifurcation diagram with $q=11$ without considering ferroresonance limiter effect.

required is easily calculated to a reasonable approximation by dividing the rated phase voltage by the rated phase current of the transformer. Ferroresonance limiter impedance is conventionally achieved using resistors rather than inductors, so as to limit the tendency for the fault arc to persist due to inductive energy storage. These resistors will dissipate considerable heat when earth fault current flows and are usually only short term rated, so as to achieve an economic design. Due to the explanation above, In Figure 6, $R_{F L R}$ is the ferroresonance limiter resistance. Typical values for various system parameters is considered for simulation were kept the same by the case 1, while ferroresonance limiter impedance is added to the power system configuration and its value is given below:

$$
R_{F L R}=50 \mathrm{~K} \Omega
$$

Typical values for various system parameters considered for simulation with ferroresonance limiter are as given in the Table 3 .

The differential equations of the equivalent circuit as shown in Figure 6 are given as bellowed:

$$
\begin{gathered}
E=v_{c}+v_{l}+v_{R_{F L R}} \\
v_{R n}=R_{F L R} \cdot i_{R_{F L R}}=R_{F L R} \cdot\left(\frac{1}{R} \frac{\mathrm{d} \varphi}{\mathrm{d} t}+a \varphi+b \varphi^{q}\right) \\
\frac{\mathrm{d} v_{l}}{\mathrm{~d} t}=\left(\frac{R}{R_{F L R}+R}\right)\left(\frac{\mathrm{d} E}{\mathrm{~d} t}-\frac{1}{R C} \frac{\mathrm{d} \varphi}{\mathrm{d} t}-\frac{1}{C}\left(a \varphi+b \varphi^{q}\right)\right. \\
\left.-R_{F L R} \cdot a \frac{\mathrm{d} \varphi}{\mathrm{d} t}-R_{F L R} q b \varphi^{q-1} \frac{\mathrm{d} \varphi}{\mathrm{d} t}\right)
\end{gathered}
$$

where, $\varphi$ is flux linkage and $v_{l}$ is the voltage of the power transformer.

\section{Simulation Results of the Power Transformer Considering Ferroresonance Limiter Effect}

Figures 7(a) and (b) show the phase plan diagram, and

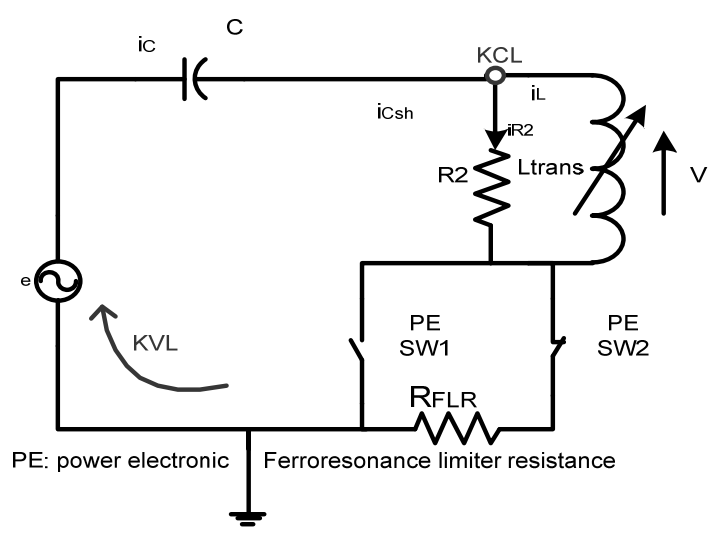

Figure 6. Equivalent circuit of the power system connecting ferroresonance limiter impedance.

Table 2. Simulation results without considering ferroresonance limiter.

\begin{tabular}{ccccccccc}
\hline$q / E$ & 1 & 2 & 3 & 4 & 5 & 6 & 7 & 8 \\
\hline 7 & P1 & P3 & P5 & chaos & chaos & chaos & chaos & chaos \\
11 & P1 & P1 & chaos & chaos & P3 & P3 & chaos & chaos \\
\hline
\end{tabular}

P: period.

Table 3. Power system parameters and per unit values.

\begin{tabular}{ccc}
\hline$Q$ & 7 & 11 \\
\hline Coefficient (a) & 0 & 0.0028 \\
Coefficient (b) & 0.001 & 0.0072 \\
$\omega$ & $377 \mathrm{rad} / \mathrm{sec}$ & $377 \mathrm{rad} / \mathrm{sec}$ \\
& $1 \mathrm{p.u}$ & $1 \mathrm{p} . \mathrm{u}$ \\
$E$ & $110 / 44 \mathrm{kv}$ & $110 / 44 \mathrm{kv}$ \\
& $1 \mathrm{p.u}$ & $1 \mathrm{p} . \mathrm{u}$ \\
$C$ & $777 \mathrm{nf}$ & $777 \mathrm{nf}$ \\
$S$ & $0.82 \mathrm{p} . \mathrm{u}$ & $0.82 \mathrm{p.u}$ \\
& $25 \mathrm{MVA}$ & $25 \mathrm{MVA}$ \\
$R_{F L R}$ & $50 \mathrm{~K} \Omega$ & $50 \mathrm{~K} \Omega$ \\
& $10 \mathrm{p} . \mathrm{u}$ & $10 \mathrm{p} . \mathrm{u}$ \\
\hline
\end{tabular}

FFT of the power system behavior. It is shown that chaotic region is controlled by applying ferroresonance limiter, and tendency to the chaos behavior also decreased.

By comparing these plots with Figures 4(a) and (b), it can be concluded that considering ferroresonance limiter effect can cause ferroresonance drop out. By increasing the degree of $q$, there is no significant change in the system behavior. Figure 8(a) shows the system overvoltage in the case of $q=11$.

Phase plan diagram that is shown in Figure 8(a) indicate the fundamental resonance of the power system with period II oscillation. This case of the simulation is shown effect of considering ferroresonance limiter on controlling nonlinear phenomena in the power transformer. Amplitude of the oscillations is 2.2 p.u, and chaotic ferroresonance of the previous case is changed to the periodic 


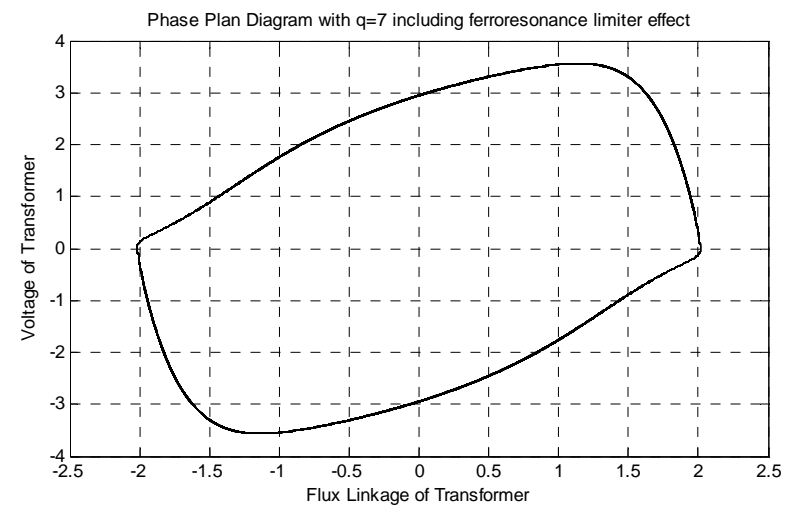

(a)

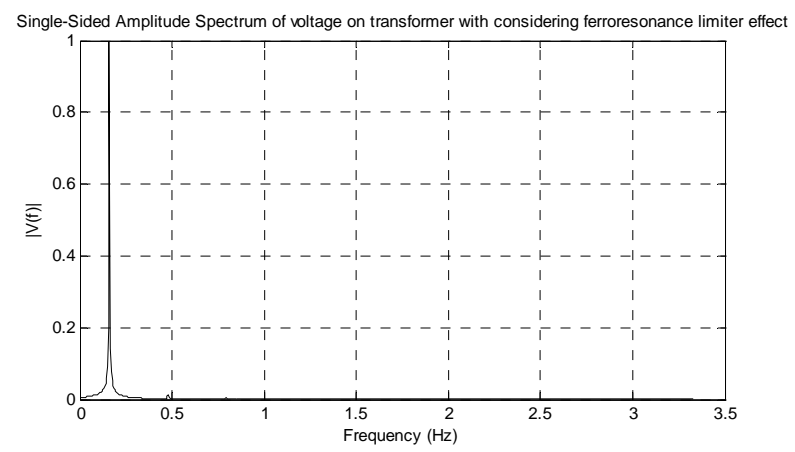

(b)

Figure 7. (a) Phase plan diagram with $q=7$ considering ferroresonance limiter effect. (b) Single-sided amplitude spectrum considering ferroresonance limiter effect.

resonance by connecting ferroresonance limiter resistance to the transformer.

Figure 9 is shown the period I oscillation, and amplitude of these oscillations is decreased to $1.8 \mathrm{p}$.u, while in the previous case, bifurcation diagram was shown chaotic oscillation with high amplitude of the ferroresonance overvoltage. By considering ferroresonance limiter, ferroresonance oscillations are successfully controlled, and changed to the periodic behavior as shown in Figure 9.

Figure 10 shows the power system overvoltage when degree of core index " $q$ " is 11 . According to this plot, in point (1), period III appears and in point (2) one jump is occurred, and trajectory of the power system is suddenly changed, and is gone to the $1.2 \mathrm{p} . \mathrm{u}$ value of the ferroresonance overvoltage. After this point, oscillation is remained with period one behavior, and in point (3) period 2 is appeared. Also, amplitude of the ferroresonance overvoltage is decreased successfully and reached to 1.4 p.u. By comparing the bifurcation diagram in Figures 9 and 10 by Figures 4 and 5, it is concluded that considering ferroresonance limiter can control the overvoltage. Table 4 includes the set of cases which are considered for analyzing the power system circuit including ferroresonance limiter resistance.

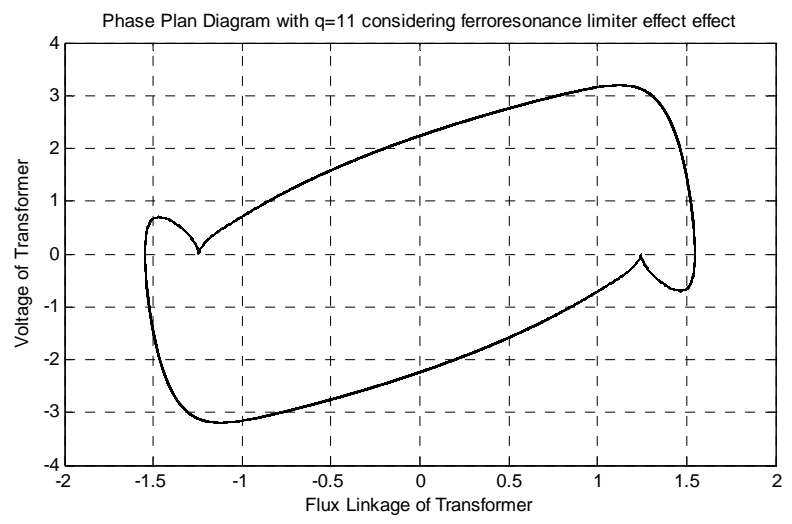

(a)

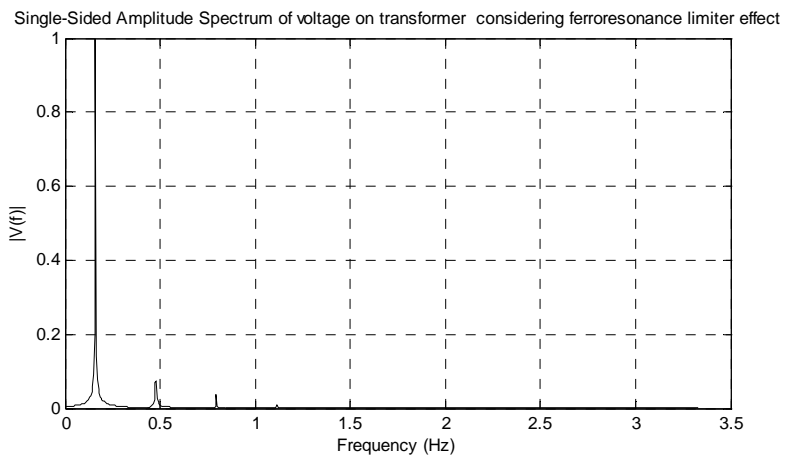

(b)

Figure 8. (a). Phase plan diagram with $q=11$ considering ferroresonance limiter effect. (b) Single-sided amplitude spectrum considering ferroresonance limiter effect.

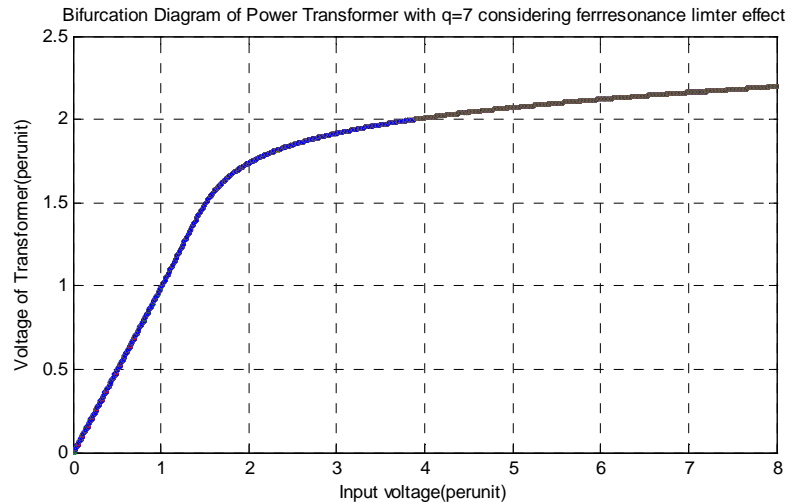

Figure 9. Bifurcation diagram with $q=7$ considering ferroresonance limiter effect.

\section{Conclusions}

In this paper, dynamic behavior of the power transformer is studied. Power transformer is modeled with linear core losses, and dynamical study clearly shows that chaotic ferroresonance can occur due to the abnormal switching action. Amplitude of these overvoltages is reached to 4 p.u. These nonlinear phenomena are very dangerous for 


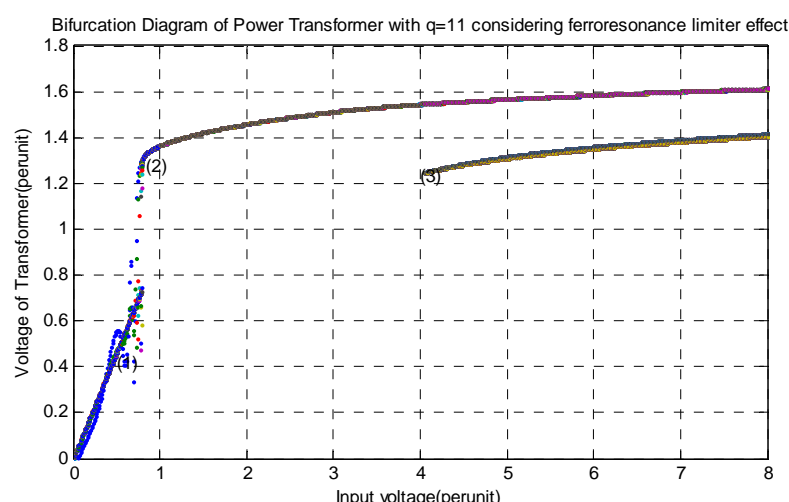

Figure 10. Bifurcation diagram with $q=11$ considering ferroresonance limiter effect.

Table 4. Simulation results considering ferroresonance limiter effect.

\begin{tabular}{ccccccccc}
\hline$q / E$ & 1 & 2 & 3 & 4 & 5 & 6 & 7 & 8 \\
\hline 7 & P1 & P1 & P1 & P1 & P1 & P1 & P1 & P1 \\
11 & P3 & P1 & P1 & P2 & P2 & P2 & P2 & P2 \\
\hline
\end{tabular}

P: period.

the power system equipment's, and specially can cause transformer failure. Also chaotic ferroresonance is controlled by considering ferroresonance limiter. Linear core losses are the most important factor for occurring ferroresonance. By considering ferroresonance limiter effect, ferroresonance overvoltage is ignored and even if unwanted phenomena are appeared, transformer can works in the safe operation region and there is no dangerous condition in the power system.

\section{References}

[1] B. A. Mork and D. L. Stuehm, "Application of Nonlinear Dynamics and Chaos to Ferroresonance in Distribution Systems," IEEE Transactions on Power Delivery, Vol. 9, No. 2, 1994, pp. 1009-1017. doi:10.1109/61.296285

[2] J. Bethenod, "Sur le Transformateur et Résonance," L'Eclairae Electrique, Vol. 30, 1907, pp. 289-296.

[3] J. W. Butler and C. Concordia, "Analysis of Series Capacitor Application Problems," AIEE Transactions, Vol. 56, No. 8, 1937, pp. 975-988.

[4] Z. Emin, B. A. T. Al Zahawi and Y. K. Tong, "Voltage Transformer Ferroresonance in $275 \mathrm{kV}$ Substation," 11th International Symposium on High Voltage Engineering, Vol. 1, No. 2, 1999, pp. 283-286.

[5] R. G. Andrei and B. R. Halley, "Voltage Transformer Ferroresonance from an Energy Transfer Standpoint," IEEE Transactions on Power Delivery, Vol. 4, No. 3, 1989, pp. 1773-1778. doi:10.1109/61.32672

[6] Y. G. Li, W. Shi, R. Qin and J. L. Yang, “A Systematical
Method for Suppressing Ferroresonance at Neutral-Grounded Substations," IEEE Transactions on Power Delivery, Vol. 18, No. 3, July 2003, pp. 1009-1014. doi:10.1109/TPWRD.2003.813858

[7] C. Charalambous, Z. D. Wang, M. Osborne and P. Jarman, "Sensitivity Studies on Power Transformer Ferroresonance of a $400 \mathrm{kv}$ Double Circuit," IET Journal of Generation, Transmission \& Distribution, Vol. 2, No. 2, March 2008, pp. 159-166.

[8] Y. G. Li, W. Shi and F. R. Li, "Novel Analytical Solution to Fundamental Ferroresonance-Part I: Power Frequency Excitation Characteristic," IEEE Transactions on Power Delivery, Vol. 21, No. 2, April 2006, pp. 788-793. doi:10.1109/TPWRD.2005.859303

[9] D. A. N. Jacobson, P. W. Lehn and R. W. Menzies, "Stability Domain Calculations of Period-1 Ferroresonance in a Nonlinear Resonant Circuit," IEEE Transactions on Power Delivery, Vol. 17, No. 3, 2002, pp. 865-871. doi:10.1109/TPWRD.2002.1022816

[10] G. Mokryani and M.-R. Haghifam, "Application of Wavelet Transform and MLP Neural Network for Ferroresonance Identification," IEEE Conference of Conversion and Delivery of Electrical Energy in the 21st Century, Pittsburgh, 20-24 July 2008, pp. 1-6, 20-24.

[11] A. Rezaei-Zare, R. Iravani and M. Sanaye-Pasand, "Impacts of Transformer Core Hysteresis Formation on Stability Domain of Ferroresonance Modes," IEEE Transactions on Power Delivery, Vol. 24, No. 1, 2009, pp. 177 186. doi:10.1109/TPWRD.2008.2002668

[12] C. A. Charalambous, Z. D. Wang, P. Jarman and M. Osborne, "2-D Finite-Element Electromagnetic Analysis of an Autotransformer Experiencing Ferroresonance," IEEE Transactions on Power Delivery, Vol. 24, No. 3, July 2009, pp. 1275-1283. doi:10.1109/TPWRD.2009.2016629

[13] P. G. Khorasani and A. Deihimi, "A New Modeling of Matlab Transformer for Accurate Simulation of Ferroresonance," International Conference on Power Engineering, Energy and Electrical Drives, Vol. 1, No. 3, March 2009, pp. 529-534.

[14] H. Radmanesh and M. Rostami, "Effect of Circuit Breaker Shunt Resistance on Chaotic Ferroresonance in Voltage Transformer," Advances in Electrical and Computer Engineering, Vol. 10, No. 3, 2010, pp. 71-77.

[15] H. Radmanesh, "Controlling Ferroresonance in Voltage Transformer by Considering Circuit Breaker Shunt Resistance Including Transformer Nonlinear Core Losses Effect," International Review on Modeling and Simulations, Vol. 3, No. 5, Part A, 2010, pp. 988-996.

[16] H. Radmanesh, "Controlling Chaotic Ferroresonance Oscillations in Autotransformers Including Linear and Nonlinear Core Losses Effect," International Review of Electrical Engineering, Vol. 5, No. 6, 2010, pp. 2644- 2652.

[17] S. Mozaffari, M. Sameti and A. C. Soudack, "Effect of Initial Conditions on Chaotic Ferroresonance in Power Transformers," IEE Proceedings*/Generation, Transmission and Distribution, Vol. 144, 1997, pp. 456-460. doi:10.1049/ip-gtd:19971459 